\title{
Tyrosinemia Type I
}

National Cancer Institute

\section{Source}

National Cancer Institute. Tyrosinemia Type I. NCI Thesaurus. Code C98641.

Tyrosinemia caused by mutations in the FAH gene. It is characterized by deficiency of the enzyme fumarylacetoacetate hydrolase. It is the most severe form of tyrosinemia. Signs and symptoms appear early in life and include failure to thrive, vomiting, diarrhea, jaundice, and bleeding tendency. It may result in liver and kidney failure. 